\title{
CORRELAÇÃO DE DADOS DE EQUILÍBRIO LÍQUIDO-VAPOR PARA SISTEMAS AQUOSOS COM ÁLCOOL E SAL USANDO O MODELO UNIQUAC ORIGINAL
}

\author{
J.A. OLIVEIRA ${ }^{1}$, E. L. FOLETTO ${ }^{2, *}$, O. CHIAVONE-FILHO ${ }^{1}$ \\ ${ }^{1}$ Universidade Federal do Rio Grande do Norte, Departamento de Engenharia Química, UFRN \\ ${ }^{2}$ Universidade Federal de Santa Maria, Departamento de Engenharia Química, UFSM \\ *E-mail: efoletto@gmail.com
}

\begin{abstract}
RESUMO - Neste trabalho é aplicada a correlação de dados de equilíbrio líquido-vapor de sistemas aquosos contendo $\mathrm{NaCl}$ e um álcool (metanol ou etanol), usando o modelo UNIQUAC e na sua forma original. Uma coleção dos dados experimentais da literatura foi levantada, permitindo uma avaliação de consistência termodinâmica e estimação de parâmetros com informações selecionadas para os dois sistemas ternários estudados a baixas pressões. O método da pressão total de Barker foi aplicado e a pressão de vapor do $\mathrm{NaCl}$ foi desprezada. A linguagem Python foi aplicada com as bibliotecas matemáticas de regressão não-linear, recursos de interface gráfica com diagramas e opções que viabilizam a estimativa de parâmetros. Os resultados para as correlações para os dois sistemas com metanol e etanol demonstraram ajustes com desvios próximos às incertezas experimentais.
\end{abstract}

\section{INTRODUÇÃO}

Os fenômenos e propriedades envolvidas nos processos químicos atuais geralmente são comportamentos não-lineares. Portanto, é necessário um método robusto de regressão não-linear para proporcionar uma correlação exigida para os dados experimentais coletados, quer a partir do laboratório ou da indústria. Bard (1974) apontou três métodos de estimativa de parâmetros, ou seja, mínimos quadrados, máxima verossimilhança e Bayesiana. O método dos mínimos quadrados é o procedimento de estimação mais antigo e mais usado. Este pode ser aplicado diretamente a um modelo determinístico, sem qualquer conhecimento em consideração à distribuição de probabilidade das observações. Esta função satisfaz muito bem à proposta de correlacionar a maioria das propriedades termodinâmicas desejadas e variáveis de processo. Para resolver estes problemas sem restrições, o método de Levenberg-Maquardt provou resultados muito confiáveis. Este método de estimativa de parâmetros tem sido reproduzido e devidamente testado para a correlação de dados de equilíbrio líquido-vapor de substâncias puras e misturas. Para o último caso, uma avaliação da consistência termodinâmica dos dados pode ser fornecida, uma vez que os modelos de coeficiente de atividade aplicados correspondem à definição exata da energia de Gibbs parcial molar em excesso. Dados de equilíbrio líquido-vapor para sistemas de solventes de eletrólitos mistos também foram considerados para a correlação. Neste trabalho, foi proposta uma nova forma para determinar os parâmetros estruturais UNIQUAC dos íons, que é compatível com os parâmetros de espécies de solventes. Kikic et al. (1991) também aplicaram a equação do coeficiente de atividade convencional para sistemas de solventes misturados com eletrólitos com o modelo de grupo funcional, ou seja, UNIFAC. Levou-se em conta o termo eletrostático com o termo de Debye-Hückel, normalizados de acordo com a teoria de Mc-Millan-Mayer. No entanto, os parâmetros estimados apresentam 


\section{9 a 22 de outubro de 2014 \\ Florianópolis/SC}

espalhamento e alta ordem de magnitude. Loehe e Donohue (1997) descrevem um levantamento dos modelos da literatura para determinar as propriedades termodinâmicas de sistemas aquosos com eletrólitos fortes. Thomsen et al. (2004) apresentaram um trabalho de modelagem sistemática com eletrólitos mistos e misturas de solventes para dados de equilíbrio sólido-líquido-vapor, usando também o modelo UNIQUAC. Haghtalab e Peyvandi (2009) propuseram uma nova versão do modelo UNIQUAC para soluções de eletrólitos que requer apenas dois parâmetros ajustados de interação por sistema aquoso binário. Os parâmetros estruturais das espécies iônicas foram avaliados através de solvatação para a determinação dos raios iônicos.

Este estudo teve como objetivo descrever o procedimento computacional Levenberg (1944) e Marquadt (1963) para a estimativa dos parâmetros do modelo de equilíbrio líquido-vapor. Aplicação de eletrólitos fortes em sistemas de solventes misturados foi também realizado com uma forma compatível para avaliar os parâmetros estruturais, utilizando-se os valores de água como referência.

\section{MÉTODOS DE CÁLCULO}

A fim de demonstrar a aplicabilidade do método de Levenberg-Marquardt implementado, códigos computacionais em Python foram desenvolvidos para correlacionar dados de equilíbrio líquido-vapor de substâncias puras e misturas binárias.

O primeiro programa permite a correlação de dados de pressão e de temperatura para os componentes puros de vapor, usando a equação de Antoine. Na Equação $1, P^{\text {Sat }}$ é a pressão de vapor, $T$ é a temperatura de saturação, e $A, B$ e $C$ são as constantes de Antoine ajustadas. Esta forma da equação de Antoine é habitual na literatura, no entanto, o utilizador aplica uma versão modificada da correlação em termos da base e unidades de logaritmo.

$$
\log _{10} P^{\text {sat }}(m m H g)=A-\frac{B}{T\left({ }^{o} C\right)+C}
$$

Para executar os dados de regressão, a função objetivo sequência $(F O)$ deve ser minimizada, a qual é o resíduo definido pela diferença absoluta entre as pressões experimentais e calculadas.

$$
F O=M I N=\sum_{i}\left(P_{e x p}-P_{c a l}\right)^{2}
$$

O programa demanda uma estimativa inicial dos parâmetros $A, B$ e $C$ a serem estimados, um valor inicial do parâmetro de Marquardt e, finalmente, um arquivo de entrada. Este arquivo deve conter o nome da substância, o número de pontos de dados e a lista de pares experimentais de pressão de vapor e da temperatura de saturação. Indicação das unidades e a fonte dos dados da literatura que têm-se recuperado. Também é importante ser registrado no campo do nome. Um arquivo de saída é criado, onde as informações sobre as interações realizadas durante a execução é registrado, permitindo a identificação e detecção de um eventual erro. Após a convergência, os valores das 
constantes são mostrados junto com o desvio padrão e a matriz de correlação dos parâmetros. O programa também fornece os desvios relativos e absolutos em termos de pressão de vapor e da estimativa do ponto normal de ebulição, a $760 \mathrm{mmHg}$, utilizando-se a correlação obtida. A correlação Antoine realizada está ilustrada na Figura 1, para o caso do acetato de propila.

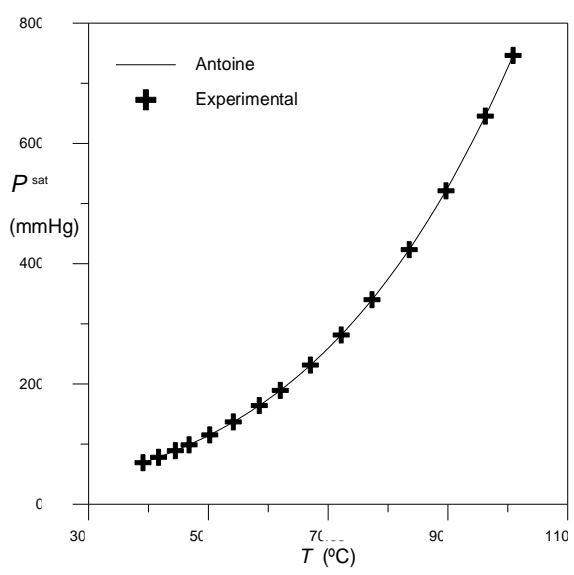

Figura 1 - Curva da pressão de vapor do acetato de propila: dados experimentais fornecidos por Boublik et al. (1984) e correlação de Antoine determinada neste trabalho; parâmetros estimados e intervalos de confidência: $A=7,03008 \pm 0,03391, B=1290,200 \pm 19,505$ e $C=209,466 \pm 2,161$

O segundo programa desenvolvido correlaciona dados de ELV de misturas binárias usando modelos da literatura (Prausnitz et al. 1999), isto é, Margules 2-sulfixos, Margules 3-sulfixos, Van Laar, Wilson e NRTL (Non-Random Two Liquids). Logo, a regressão dos dados consiste na correspondente estimativa dos parâmetros dos modelos de coeficiente de atividade $\left(\gamma_{\mathrm{i}}\right)$. No caso de dados completos de ELV, a correlação avalia também a consistência termodinâmica dos dados, ou seja, teste dos desvios (Fredenslund et al., 1977). Isto se deve ao fato de que modelos de coeficiente de atividade satisfazem a definição exata da energia livre de Gibbs de excesso parcial molar.

$$
\ln \gamma_{i} \equiv\left[\frac{\partial\left(n G^{E} / R T\right)}{\partial n_{i}}\right]_{T, P, n_{i \neq j}}=\frac{\bar{G}_{i}^{E}}{R T}
$$

A correlação dos dados de ELV de um sistema binário pode ser descrita pela equação da pressão total (Barker, 1953), desprezando as não idealidades da fase vapor e a correção de Poyinting, simplificações adequadas para baixas pressões e componentes não associáveis.

$$
P=x_{1} \gamma_{1} P_{1}^{s a t}+\left(1-x_{1}\right) \gamma_{2} P_{2}^{s a t}
$$


Deve ser destacado que os coeficientes de atividade dependem da composição da fase líquida, da temperatura e dos seus parâmetros, os quais são estimados na regressão dos dados. Para dar uma ilustração, a equação de Margules 2-sufixos é descrita a seguir para as espécies 1 e 2.

$$
\ln \gamma_{1}=A x_{2}^{2} ; \quad \ln \gamma_{2}=A x_{1}^{2}
$$

Logo, a redução dos dados da mistura é similar à correlação de Antoine e pode também ser aplicada a função objetivo representada pela Equação 2. Após a regressão, a composição da fase vapor pode ser avaliada pela Equação 6.

$$
y_{1, c a l}=\frac{\gamma_{1} x_{1} P_{1}^{s a t}}{x_{1} \gamma_{1} P_{1}^{s a t}+\left(1-x_{1}\right) \gamma_{2} P_{2}^{s a t}}
$$

Os dados de equilíbrio líquido-vapor definidos para o sistema $n$-heptano(1) + etil butirato (2), a $100{ }^{\circ} \mathrm{C}$, apresentado por Kojima e Tochigi (1979), foi usado para testar o método de regressão nãolinear implementado. O programa desenvolvido permite a estimativa de parâmetros dos cinco modelos de coeficiente de atividade listados na Tabela 1. Observou que todos os modelos descreveram satisfatoriamente os dados experimentais. Além disso, os desvios apresentados indicam que os dados experimentais são consistentes. Após a convergência, os valores dos parâmetros estimados são apresentados junto com a matriz de correlação, os desvios, os parâmetros estimados e os respectivos intervalos de confidência.

Tabela 1 - Correlação e teste de consistência dos dados de equilíbrio vapor-líquido para o $n$ heptano(1) + etil butirato (2), à $100^{\circ} \mathrm{C}$ (Kojima e Tochigi, 1979).

\begin{tabular}{lllll}
\hline Model & $A$ & $B$ & $\Delta P(\%)$ & $\Delta y_{1}^{* * *}$ \\
\hline Margules 2-sufixos & 0,5563 & & 1,36 & 0,0057 \\
Margules 3- sufixos & 0,7486 & 0,4059 & 0,87 & 0,0097 \\
Van Laar & 0,4664 & 0,5769 & 0,76 & 0,0096 \\
Wilson & 0,3928 & 1,1612 & 1,02 & 0,0089 \\
NTRL $^{*}$ & 1,2171 & $-0,4020$ & 0,98 & 0,0091 \\
\hline
\end{tabular}

* $C$ é o parâmetro randômico e foi usado o valor de 0,3 como valor padrão.

** Os valores do desvio médio absoluto são menores do que $0,01\left(\Delta \mathrm{y}_{1}<0,01\right)$, indicando que os dados são considerados consistentes, segundo Fredenslund et al. (1977).

\section{RESULTADOS E DISCUSSÃO}

O método de Levenberg-Marquart foi aplicado para duas misturas aquosas ternárias com etanol e $\mathrm{NaCl}$ e metanol e $\mathrm{NaCl}$. Os correspondentes sistemas binários também foram correlacionados. $\mathrm{O}$ modelo de coeficiente de atividade UNIQUAC (Abrams e Prausnitz, 1975) foi usado considerando o $\mathrm{NaCl}$ como uma espécie molécular, ou seja, desprezando a dissociação do sal. A Tabela 2 apresenta os parâmetros estruturais utilizados para as espécies estudadas. Deve-se salientar que os parâmetros estruturais das espécies de sal, isto é, parâmetros de área superficial e volume foram determinados a 


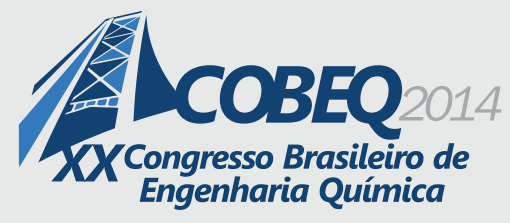

partir dos valores da água e uma razão de massa molecular. Dois objetivos foram alcançados usando essa abordagem. Um deles foi fornecer o ajuste dos dados com uma razoável ordem de grandeza dos parâmetros estruturais. Em segundo lugar, encontrar a compatibilidade com os parâmetros de interação estimados para misturas binárias de não-eletrólitos. Esta é também uma característica importante para a previsibilidade do modelo e de aplicações de contribuição de grupos, tais como o modelo UNIFAC (Kikic et al., 1991). Os valores de $r$ e $q$ para espécies iônicas foram ajustados por Macedo et al. (1990 ). Outra abordagem encontrada na literatura é o incremento dos parâmetros do raio iônico, pela consideração da solvatação. No entanto, o uso destes dois métodos exige a estimativa de novos parâmetros de solvente-solvente e, assim, a necessidade de compatibilidade não é cumprida. Por outro lado, utilizando-se os valores de $r$ e $q$ calculados a partir dos valores de água e uma razão de massas molaculares, como apresentado na Tabela 2. Foi obtida a compatibilidade desejada e, simultaneamente, uma boa capacidade de correlação, eliminando o problema de baixos valores estruturais, devido ao tamanho do íon para equação UNIQUAC.

A Tabela 3 apresenta os parâmetros de interação UNIQUAC estimados para as espécies estudadas. Deve-se salientar que os sistemas binários não-eletrólitos têm sido correlacionados inicialmente e separadamente e, em seguida, com estes valores predeterminados, foram estimadas as constantes de sal-solvente a partir dos dados experimentais correspondentes. Todos os cálculos apresentaram desvio médio absoluto em termos de fração molar de vapor menor que 0,01 e desvio relativo médio em termos de pressão menor de $2 \%$. Estes desvios indicam a consistência dos dados experimentais e, também, a qualidade dos parâmetros que reunem muitos pontos experimentais e de diferentes fontes.

Tabela 2 - Parâmetros estruturais para o modelo UNIQUAC.

\begin{tabular}{|c|c|c|c|c|}
\hline Espécies $i$ & Metanol & Etanol & Água & $\mathrm{NaCl}$ \\
\hline$r_{\mathrm{i}}$ & 1,4311 & 2,1055 & 0,9200 & $2,9845^{\mathrm{a}}$ \\
\hline$q_{\mathrm{i}}$ & 1,4320 & 1,9720 & 1,4000 & $4,5417^{b}$ \\
\hline
\end{tabular}

Tabela 3 - Estimativa dos parâmetros de interação UNIQUAC $\left(a_{\mathrm{ij}}\right)$ em Kelvin para os sistemas estudados com metanol $(\mathrm{MeOH})$, etanol $(\mathrm{EtOH})$, água $\left(\mathrm{H}_{2} \mathrm{O}\right)$ e cloreto de sódio $(\mathrm{NaCl})$.

\begin{tabular}{ccccc}
\hline$i \backslash j$ & $\mathrm{MeOH}$ & $\mathrm{EtOH}$ & $\mathrm{H}_{2} \mathrm{O}$ & $\mathrm{NaCl}$ \\
\hline $\mathrm{MeOH}$ & 0,00 & $432,85^{\mathrm{a}}$ & $-103,32^{\mathrm{b}}$ & $-932,37^{\mathrm{d}}$ \\
$\mathrm{EtOH}$ & $-261,57^{\mathrm{a}}$ & 0,00 & $-12,47^{\mathrm{c}}$ & $-878,07^{\mathrm{d}}$ \\
$\mathrm{H}{ }_{2} \mathrm{O}$ & $150,07^{\mathrm{b}}$ & $166,12^{\mathrm{c}}$ & 0,00 & $-925,33^{\mathrm{d}}$ \\
$\mathrm{NaCl}$ & $5817,00^{\mathrm{d}}$ & $4722,55^{\mathrm{d}}$ & $53,67^{\mathrm{d}}$ & 0,00 \\
\hline
\end{tabular}

a Parâmetros estimados dos dados de ELV metanol+etanol (Niesen et al., 1986; Kurihara et al., 1993).

aParâmetros estimados dos dados de ELV metanol+água (Soujanya et al., 2010; Yao et al., 1999; Kurihara et al., 1993).

'Parâmetros estimados dos dados de ELV etanol+ água (Pemberton e Mash, 1978; Niesen et al., 1986; Kurihara et al., 1993, 1995; Arce et al., 1996; Navarro-Espinosa et al., 2010; Lai et al. 2014).

'Parâmetros estimados dos dados de ELV água+NaCl, metanol+água+NaCl e etanol+água+NaCl (Clarke e Glew, 1985; Gmehling, 1997; Yang e Chul et al., 1998; Jödecke et al., 2005), fixando os parâmetros $\mathrm{MeOH}+\mathrm{EtOH}, \mathrm{MeOH}+\mathrm{H}_{2} \mathrm{O}$ e EtOH$+\mathrm{H}_{2} \mathrm{O}$, determinados previamente a partir dos binários ${ }^{\mathrm{a}, \mathrm{b}, \mathrm{c}}$. 
As Figuras 2 e 3 ilustram a qualidade da correlação obtida para os sistemas ternários aquosas com metanol e $\mathrm{NaCl}$ a 314,6 K e com etanol e $\mathrm{NaCl}$ a $700 \mathrm{~mm} \mathrm{Hg}$, respectivamente.

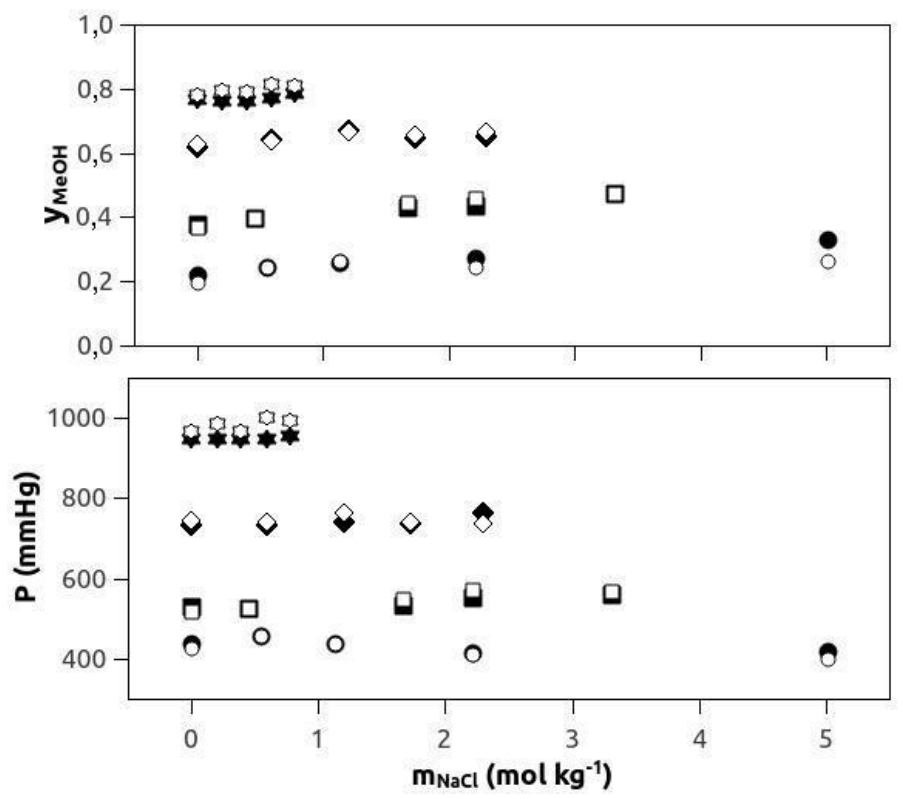

Figura 2 - Diagrama de ELV para metanol(1)+água(2)+NaCl(3) a 314,6 K; símbolos sólidos: experimental (Jödecke et al., 2005) e símbolos abertos: modelo UNIQUAC (neste trabalho). $\bigcirc$ e $\bigcirc$, $x_{1}^{\prime}=0,0328 ; \boldsymbol{\Delta}$ e $\triangle, x_{1}^{\prime}=0,0789 ; \mathbf{\square} \square, x_{1}^{\prime}=0,216 ; \nabla$ e $\nabla, x_{1}^{\prime}=0,466$.

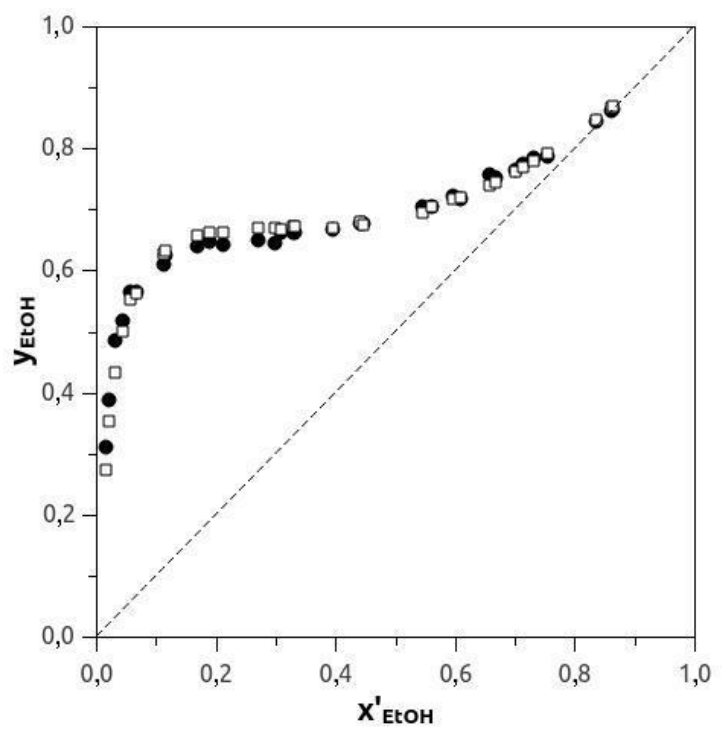

Figura 3 - Diagrama $y$ - $x$ do ELV para sistema etanol(1)+água(2)+NaCl(3) a $700 \mathrm{mmHg}$; 32 pontos experimentais com fração molar do sal na faixa de 0,001-0,1; dados experimentais (Gmehling, 1997); $\square$ valores calculados com UNIQUAC (neste trabalho). 


\section{9 a 22 de outubro de 2014 \\ Florianópolis/SC}

\section{CONCLUSÕES}

A coerência dos programas desenvolvidos foi verificada através de comparação com os parâmetros estimados para o mesmo conjunto de dados, confirmando a confiabilidade das rotinas implementadas de Levenberg-Marquardt. Os resultados do programa de correlação para as misturas foram eficientes e a análise dos desvios permitiu a avaliação da consistência termodinâmica dos dados experimentais. A ferramenta desenvolvida de cálculo poderia ser aplicado em problemas de simulação e otimização de processos, utilizando inclusive os recursos visuais, o qual facilitaria a interação com os usuários. Além disso, os novos programas de correlação também poderiam ser desenvolvidos para descrever outras propriedades termodinâmicas e físicas-químicas, uma vez que a rotina Levenberg-Marquardt tenha sido adequadamente implementada. Estes novos parâmetros estruturais das espécies de sal demonstraram também serem flexíveis para a correlação de dados e coerência em relação à ordem de grandeza. A contribuição eletrostática não foi considerada nos cálculos de ELV. A forma original do modelo UNIQUAC demonstrou ser viável com a abordagem molecular de espécies de eletrólitos fortes em sistemas de solventes mistos.

\section{AGRADECIMENTOS}

ANP, Petrobras, CAPES, CNPq e INCT-EMA.

\section{REFERÊNCIAS}

ABRAMS, D.S.; PRAUSNITZ, J.M. Statistical thermodynamics of liquid mixtures: a new expression for the excess Gibbs energy of partly or completely miscible systems. AIChE J., 21, 116128,1975 .

ARCE, A.; MARTÍNEZ-AGEITOS, J.; SOTO, A. VLE for water + ethanol + 1-octanol mixtures. Experimental measurements and correlations. Fluid Phase Equil., 122, 117-129, 1996.

BARD, Y. Nonlinear parameter estimation. New York: Academic Press; 1974.

BARKER, J.A. Determination of activity coefficients from pressure total measurements. Austral. $J$. Chem., 6, 207-210, 1953.

BOUBLIK ,T.; FRIED, V.; HÁLA, E. The vapor pressure of pure Substances. Amsterdam: Elsevier; 1984.

CLARKE, E.C.; GLEW, D.N. Evaluation of the Thermodynamic Functions for Aqueous Sodium Chloride from Equilibrium and Calorimetric Measurements below $154^{\circ} \mathrm{C}$. J. Phys. Chem. Ref. Data, 14, 2, 489-610, 1985.

FREDENSLUND, A.; GMEHLING, J.; RASMUSSEN, P. Vapor-liquid equilibria using UNIFAC. Amsterdan: Elsevier; 1977.

HAGHTALAB, A.; PEYVANDI, K. Electrolyte-UNIQUAC-NRF model for the correlation of the mean activity coefficient of electrolyte solutions. Fluid Phase Equil., 281, 163-171, 2009.

GMEHLING, J. Dortmund Data Bank (DDB) - electrolytes (ELE). Oldenburg, Germany: DDBST $\mathrm{GmbH}, 1997$.

JÖDECKE, M.; KAMPS, A.P.S.; MAURER, G. Experimental Investigation of the Influence of NaCl 


\section{9 a 22 de outubro de 2014 \\ Florianópolis/SC}

on the Vapor-Liquid Equilibrium of $\mathrm{CH}_{3} \mathrm{OH}+\mathrm{H}_{2} \mathrm{O}$. J. Chem. Eng. Data, 50, 1, 138-141, 2005.

KIKIC, I.; FERMEGLIA, M.; RASMUSSEN, P. UNIFAC prediction of vapor-liquid equilibria in mixed solvent-salt systems. Chem. Eng. Sci., 46, 2775-2780, 1991.

KOJIMA, K.; TOCHIGI, K. Prediction of Vapor-Liquid Equilibria by the ASOG. Amsterdam: Elsevier, 1979.

KURIHARA, K.; NAKAMICHI, M.; KAZUO K. Isobaric vapor-liquid equilibria for methanol + ethanol + water and the three constituent binary systems. J. Chem. Eng. Data, 38, 3, 446-449, 1993.

KURIHARA, K.; MINOURA, T.; TAKEDA K.; KAZUO, K. Isothermal Vapor-Liquid Equilibria for Methanol + Ethanol + Water, Methanol + Water, and Ethanol + Water. J. Chem. Eng. Data, 40, 3, 679-684, 1995.

LAI, H.S.; LIN, Y.; CHEIN-HSIUN, T. Isobaric (vapor + liquid) equilibria for the ternary system of (ethanol + water $+1,3$-propanediol) and three constituent binary systems at $\mathrm{P}=101.3 \mathrm{kPa} . J$. Chem. Thermod., 68, 13-19, 2014.

LEVENBERG, K. A method for the solution of certain nonlinear problems in least squares. Quart. Appl. Math., 2, 164-168, 1944.

LOEHE, J.R.; DONOHUE, M.D., Recent advances in modeling thermodynamic properties of aqueous strong electrolyte systems. AIChE J., 43, 1, 180-195, 1997.

MACEDO, E.A.; SKOVBORG, P.; RASMUSSEN, P. Calculation of phase equilibria for solutions of strong eletrolytes in solvent-water mixtures. Chem. Eng. Sci., 45, 875-882, 1990.

MAQUARDT, D.W. An algorithm for least squares estimation of nonlinear parameters. SIAM J., 11, 431-441, 1963.

NAVARRO-ESPINOSA, I.R.; CARDONA, C.A.; JIMMY, A. L. Experimental measurements of vapor-liquid equilibria at low pressure: Systems containing alcohols, esters and organic acids. Fluid Phase Equil., 287, 141-145, 2010.

NIESEN, V.; PALAVRA, A.; KIDNAY, A.J.; YESAVAGE, V.F. An apparatus for vapor-liquid equilibrium at elevated temperatures and pressures and selected results for the water-ethanol and methanol-ethanol systems. Fluid Phase Equil., 31, 3, 283-298, 1986.

PEMBERTON, R.C.; MASH, C.J. Thermodynamic properties of aqueous non-electrolyte mixtures. ii. Vapor pressures and excess Gibbs energies for water + ethanol at 303.15 to $363.15 \mathrm{~K}$ determined by an accurate static method. J. Chem. Thermodyn., v. 10, p. 867-888, 1978.

PRAUSNITZ, J.M.; LICHTENTHALER, R.N.; AZEVÊDO, E.G. Molecular thermodynamics of fluid phase equilibria. 3rd ed., Upper Saddle River: New Jersey, Prentice-Hall, 1999.

SOUJANYA, J.; SATYAVATHI, B.; PRASAD, T.E.V. Experimental (vapour + liquid) equilibrium data of (methanol + water + glycerol) and (methanol + glycerol) systems at atmospheric and subatmospheric pressures. J. Chem. Thermod., 42, 5, 621-624, 2010.

THOMSEN, K.; ILIUTA, M.C.; RASMUSSEN, P. Extended UNIQUAC model for correlation and prediction of vapor-liquid-liquid-solid equilibria in aqueous salt systems containing nonelectrolytes. Part B. Alcohol (ethanol, propanols, butanols)-water-salt systems. Chem. Eng. Sci., 59, 3631-3647, 2004.

YANG, S.; CHUL, S.L. Vapor-Liquid Equilibria of Water + Methanol in the Presence of Mixed Salts. J. Chem. Eng. Data, 43, 4, 558-561, 1998.

YAO, J.; LI, H.; SHIJUN, H. Vapor-liquid equilibrium data for methanol-water-NaCl at $45^{\circ} \mathrm{C}$. Fluid Phase Equil., 162, 2, 253-260, 1999. 